\title{
The Teaching Circle for Large Engineering Courses: Clearing the Activation Barrier
}

\section{Dr. Cynthia J. Finelli, University of Michigan}

Dr. Cynthia Finelli is Director of the Center for Research on Learning and Teaching Engineering and research associate professor in the College of Engineering at the University of Michigan. She actively pursues research in engineering education and assists other faculty at U-M in their scholarly endeavors. Her current research interests include studying faculty motivation to change classroom practices, evaluating methods to improve teaching, and exploring ethical decision-making in undergraduate engineering students. Dr. Finelli leads a national initiative to create a taxonomy/keyword outline for the field of engineering education research, and she is past Chair of the Educational Research and Methods Division of the American Society of Engineering Education.

\section{Dr. Joanna Mirecki Millunchick, University of Michigan}

Joanna Mirecki Millunchick is a professor of materials science and engineering at the University of Michigan. She is active in scientific and pedagogical research. Her current work in education research revolves around examining student use of multimedia resources such as screencasts to improve their performance in large lecture courses. She is also the current Academic Director for M-STEM Academies, a program devoted to strengthening and diversifying the cohort of students who receive their baccalaureate degrees in science, technology, engineering, and mathematics. 


\section{The Teaching Circle for Large Engineering Courses: Clearing the Activation Barrier}

The use of active learning techniques and other student centered teaching practices in the classroom has been demonstrated by research to improve student learning and retention (e.g., $[11,12])$. In spite of this evidence, though, many faculty still primarily use the didactic lecture format in large courses, where faculty note that it can be challenging to motivate and engage students (e.g., [6]). Barriers to faculty adoption of research-based effective teaching practices include insufficient time to implement a new approach, lack of familiarity with the practices, suspicions about their efficacy, and expected student resistance (e.g., [1,7]). Our studies at University of Michigan (U-M) confirm these findings for our own engineering faculty [3, 5].

Many institutions rely on professional development programs to overcome some of these barriers, but there is ample evidence that traditional models of faculty development, primarily comprised of one-time workshops, have not resulted in widespread adoption of research-based effective teaching practices. Building on the literature for successful faculty professional development, we designed and implemented the "Teaching Circle for Large Engineering Courses," a cohort-based program for engineering faculty at our large research university to learn about effective teaching practices and to implement new teaching techniques in their own course. We show that, as a result of their involvement in this term-long program, participants approached teaching differently and were actively altering their behavior in the classroom. Thus, we succeeded in helping faculty overcome the inertia they often experience when adopting new teaching practices.

\section{Our Teaching Circle Program}

Success of a faculty professional development program is partially due its design. Programs that take into account factors for adult motivation (e.g., [14]) are likely to be more effective than those that do not [2]. For instance, the perceived expertise of the program facilitators must not be in question, and the content must be directly relevant to the interests and goals of the participants. The participants must also have a choice in how to apply the presented concepts, as well as the opportunity to put them into practice.

With these criteria in mind, we designed our "Teaching Circle for Large Engineering Courses” to inform faculty about effective teaching practices, influence their approaches to teaching, and give them the tools to change their teaching behaviors and adopt effective teaching practices in large classrooms. The program included four monthly sessions over the term, and it was cofacilitated by the authors of this paper: a senior engineering faculty member (a professor in the Materials Science and Engineering Department at U-M) and an experienced faculty developer (director of the Center for Research on Learning and Teaching in Engineering (CRLT-Engin) and research associate professor at U-M). Participants were expected to have a midterm student feedback session (a.k.a., Small Group Instructional Diagnosis, [4]) conducted in their course to evaluate the impact of their efforts. Upon completion of the program, participants were eligible for a $\$ 1,000$ grant to support their teaching in large courses. Due to staffing and budget constraints, the program was limited to seven faculty in the first offering. 
All engineering faculty U-M were invited to apply for the first offering of the Teaching Circle. Of the nearly 400 full-time faculty teaching large courses when the program was announced, 25 applied to participate. After careful deliberation, the facilitators selected seven faculty who were not regular participants in programs offered by CRLT-Engin and who collectively represented a range of rank, experience, and discipline. These seven comprise the treatment group. Eight faculty who applied but were not invited to participate and who were roughly matched to the participants agreed to serve as a control group. Because these eight faculty had applied to the Teaching Circle, we consider selection-bias to be only a minor issue. Further, at the start of the program, the attitudes and behaviors of the treatment group faculty were comparable to those of the faculty in the control group, so comparing changes in attitude and behavior over the term offers an indication of the impact of the Teaching Circle. Demographics of faculty in the treatment and control groups are listed in Table 1.

Table 1. Demographics of treatment and control groups

\begin{tabular}{llcc}
\hline & & Treatment & Control \\
Gender & $\mathbf{N = 8}$ \\
\hline \multirow{3}{*}{ Rank } & Male & 4 & 5 \\
& Female & 3 & 3 \\
\hline \multirow{5}{*}{ Department } & Assistant Professor or Lecturer & 2 & 3 \\
& Associate Professor & 2 & 0 \\
& Professor & 3 & 5 \\
\hline & Atmospheric, Oceanic, and Space Sciences & 1 & 1 \\
& Biomedical Engineering & 1 & 1 \\
& Civil and Environmental Engineering & 1 & 0 \\
& Chemical Engineering & 1 & 0 \\
& Electrical Engineering and Computer Science & 1 & 2 \\
& Industrial and Operations Engineering & 1 & 1 \\
& Mechanical Engineering & 1 & 1 \\
& Technical Communication & 0 & 2 \\
\hline
\end{tabular}

The four sessions of the Teaching Circle addressed topics selected in consultation with the participants. These include: building rapport in large classes, active learning techniques, student motivation, mis-/pre-conceptions, and instructional technology (screencasts and classroom response systems). Each session featured readings that summarized relevant research and highlighted practical strategies for success, and there was considerable discussion amongst the participants. Over the term, faculty interacted extensively with the two program facilitators, with each other, and with other senior faculty who were invited guests at meetings.

\section{Evaluation of Program Impact}

As Felder notes, only a few aspects of changes in participant behavior as a result of a faculty professional development program can realistically be measured, but determining the impact of a program on attitudes and behaviors is a more meaningful goal than merely measuring participant satisfaction [2]. Here we report three metrics we used to evaluate the success of the program. 


\section{Attitudes and behaviors}

First, we study self-reported changes in attitude and behavior. To do this, we administered Trigwell and Prosser's Approaches to Teaching Inventory (ATI, [13]) and Murray’s Teaching Behaviors Inventory (TBI, [10]) at the beginning and end of the term to both the treatment and control groups.

The ATI is a short, research-validated inventory that measures key aspects about the way an individual approaches his/her teaching. The instrument contains 22 items - eleven represent an information transmission or teacher-focused approach and eleven represent a conceptual change or student-focused approach. Instructors who primarily approach teaching as an organized way to transmit knowledge to students are content oriented, and they score higher on the teacherfocused scale. On the other hand, those who approach teaching as a way to facilitate students' construction of knowledge are learning oriented, and they score higher on the student-focused scale. For example, "I should know the answers to any questions that students may put to me during this subject" and "In this subject, students should focus their study on what I provide them" are items that address an instructor's teacher-focused approach. But, "I see teaching as helping students develop new ways of thinking in the subject" and "A lot of teaching time in this subject should be used to question students' ideas" are items that address an instructor's studentfocused approach. Research evidence has shown that where teachers adopted more studentfocused approaches to teaching, their students adopted a deeper approach to learning [9]. Respondents reported the frequency with which they adopted the 22 teaching approaches, and we averaged the 11 scores for each scale to compute an overall teacher-focused and student-focused score. Each score ranges from 1 (only rarely or never) to 5 (almost always or always).

The TBI includes 30 separate low-inference teaching behaviors categorized into six metabehaviors: enthusiasm, clarity, interaction, task orientation, rapport, and organization. For example, "Stress important points" is a specific low-inference behavior in the clarity category; "Encourage questions and comments" is in the rapport category; and "Put outline of lecture on blackboard or overhead screen" is in the category of organization. Respondents indicated the degree to which they implemented each of the 30 low-inference behaviors, and we computed an overall score for each of the five meta-behaviors on a five-point scale (from 1=almost never to $5=$ almost always). Though the TBI assesses self-reported behavior, comparisons of responses over time provide an indication of changes in behavior.

\section{Perceptions}

Second, we administered an end-of-term survey to the treatment group to assess participants' impressions of the Teaching Circle program. The survey included a series of open-ended items about participants' initial expectations of the program, whether the program met their expectations, what they found most useful, and changes they suggested for improving the program.

\section{Classroom practices}

Third, trained observers conducted classroom observations for faculty in the treatment group and a subset of the control group at the beginning and end of the term. The observers used a structured two-part observation protocol (based on Hora, Ferrare, and Anderson's work [8]) which includes items for the observer to code types of instructional method (including questions 
asked by faculty and by students), level of student engagement, cognitive activity of students, and material artifacts. We refined the protocol for another study at U-M ([3]), and here we report broad comparisons between the treatment and control group.

\section{Findings}

\section{Attitudes and behaviors}

Figure 1 shows the absolute change in teacher-focused and student-focused approach to teaching for the treatment and control group. The 95\% confidence interval is displayed, as well. The difference between the treatment and the control group is not statistically significant when comparing absolute change in teacher-focused approach (i.e., the 95\% confidence intervals for these two groups overlap). However, the absolute change is significantly greater, on average, for members in the treatment group than for the control group $(0.94 \pm 0.60$ versus $0.32 \pm 0.26$, $\mathrm{p}<.05$ ). This suggests that, as a result of participating in the Teaching Circle, instructors approached their teaching in more student-focused ways, while their teacher-focused behaviors remained nominally the same.

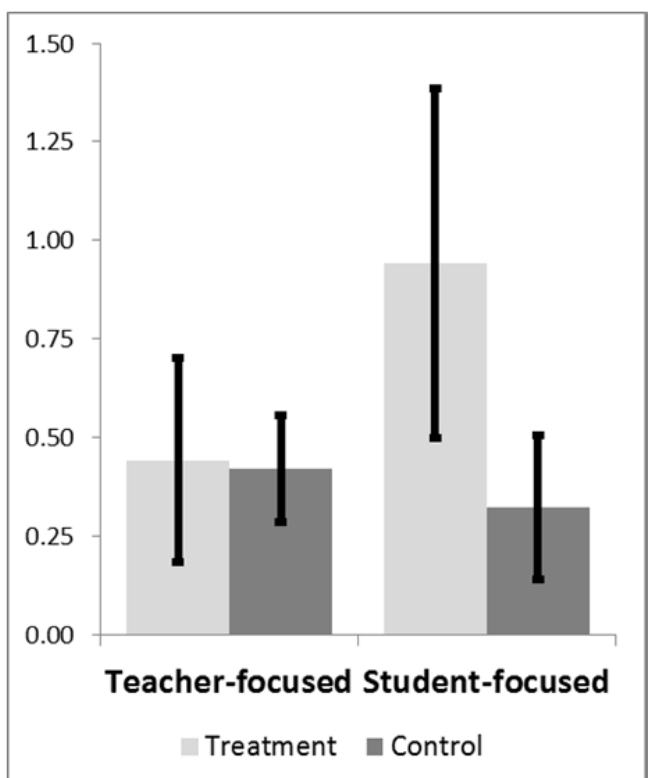

Figure 1. Absolute change in ATI scores with $95 \%$ confidence interval.

Figure 2 shows the increase in self-reported adoption of the six teaching behaviors measured by Murray's TBI for the treatment and control group. The 95\% confidence interval is displayed, as well. Scores on all six of the behaviors increased more, on average, for members in the treatment group than they did for the control group. And the control group decreased in its adoption of all but one of the behaviors (task orientation). Although most of the differences are not statistically significant, the category of enthusiasm is an exception. The treatment group had initial scores that were significantly lower than those of the control group (3.81 \pm 0.38 versus $4.33 \pm 0.56$, $\mathrm{p}<.05)$, and the gain in enthusiasm is significantly different $(0.48 \pm 0.58$ versus $0.23 \pm 0.27$, $\mathrm{p}<.05$ ). Thus, participation in the Teaching Circle is related to significant improvements in selfreported teaching behavior. 


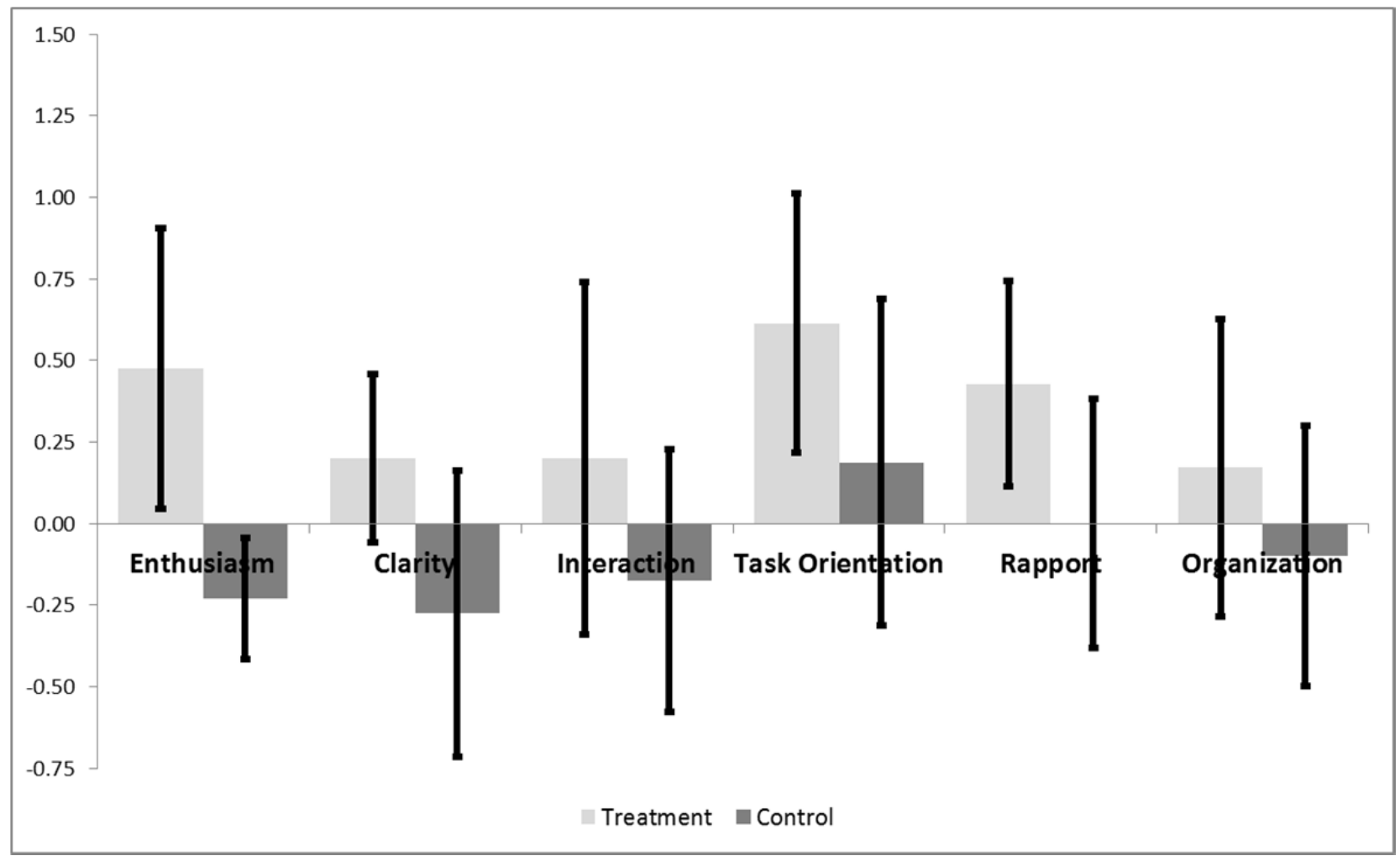

Figure 2. Increase in TBI score with $95 \%$ confidence interval.

\section{Perceptions}

While the end-of-term survey completed by participants is the most basic form of analysis possible, it provides useful insights into the perceived efficacy of the program. All of the participants stated that the reason they participated in the Teaching Circle was to learn about active learning methods appropriate for large classes, identify strategies for implementing new technologies, and interact with other faculty who value teaching. All participants also expressed that they enjoyed the program and that it met their expectations. One respondent said:

\section{It was a fantastic program that far exceeded my expectations!}

The most important outcome identified by the participants was the opportunity for meeting with like-minded faculty from other disciplines. In fact, the most common suggestion to improve the Teaching Circle was to allow for even more interaction. The participants were also asked whether or not the program affected how they approached teaching, and they all responded in the affirmative. One respondent said:

The program definitely affected how I approach teaching. I am now aware of the resources and ways I can try to use in my teaching. This is empowering to me.

Thus, according to participant perceptions, the program was successful.

\section{Classroom practices}

Classrooms for faculty in the treatment group and a subset of the control group were visited by an objective observer early and late in the term in an attempt to identify changes in classroom behavior and subsequent student engagement. A review of the consultant observation data for the 
treatment group reveals that several instructors altered their teaching behaviors in an attempt to increase student engagement and active learning with positive results, while others did not. The differences (whether positive or negative) could be characterized as incremental, in agreement with the instructors' self-assessment. Some changes in teaching include the instructor who altered an in-class activity to include a discussion about the correct answer towards the end of the term, rather than just displaying the correct answer (observed early in the term). Another instructor encouraged students to discuss the answer to quiz questions with their neighbors during the late-in-the-term class period, rather than working individually to solve the problem, as during the pre-treatment class period.

There were no clear trends for the control group, however. In some cases, there was little change in both instructor behavior and student engagement early and late in the term, while in others there were both positive and negative changes.

\section{Discussion and Summary}

Both the self-reported surveys and observer data show that instructors were more likely to change the way they approached teaching and adopt different teaching behaviors as a result of participating in the Teaching Circle. While this change was not always towards more studentfocused and active learning methods, the fact that there was a change suggests that these instructors were experimenting with new approaches in their classrooms. One participant expressed:

The possibility of changing my classroom from one of passive learning to one that incorporates some active learning is the big take-away possibility that this program provided. Plus, I now believe that I can undertake such a transition in an incremental fashion that allows me to avoid huge risky time investments and to take corrective action in timely manner

Implementing active learning approaches in the classroom can be a perilous proposition for the instructor. These approaches are often foreign to the instructor, not resonating with his/her experience as either a students or teacher, and they can be perceived as covering less content. This faculty professional development program provided participants with an opportunity to become familiar with active learning approaches and related new technologies. Furthermore, it gave them permission to gradually shift their teaching approach, as opposed to overhauling an entire course from the ground up, in the safety of a supportive community.

The format of the Teaching Circle allowed for a great deal of interaction between the facilitators and the participants, as well as between the participants themselves. This speaks to several of the criteria Wlodkowski notes for adult motivation [14], including relevance of content (because participants were helped select topics for the program), choice in application (because participants were asked to reflect on how these methods could be applied in their own classroom), praxis (because the participants had the opportunity to put these methods into practice), and group work (via the networking and community-building nature of the program). The participants also noted that the combination of an experienced faculty developer, who brought in credible research on effective teaching practices, and a respected engineering professor, a peer who validated that these practices were useful, made the Teaching Circle more powerful. In addition, the facilitators modeled the very methods they were describing by 
incorporating several active learning elements such as think-pair-share. In this way the participants experienced first hand the power of active learning. One participant said:

I think the most "fun" part of the program was the spontaneous eruption of round-table informal brainstorming sessions about our day-to-day problems, solutions, and anecdotes. You were very wise to keep it going, even though your planned agenda for material from the book, etc. was sacrificed in the process. The give and take was very enjoyable. I got a lot out of it and I think the others did, too.

The participants saw that allowing for active thinking and application of the concepts had more impact than simply presenting the material, even at the expense of other planned activities.

Additional analysis and follow up with the participants is underway to determine the long term effect of the program and the resulting impact on student learning. The program was oversubscribed in its initial offering, which compelled the college to continue funding this effort. The second Teaching Circle was recently concluded, and preliminary findings echo what we present here. A third offering is underway, and based on the continued interest of the faculty, this will likely be an ongoing program.

The term-long Teaching Circle for Large Engineering Courses helped faculty clear the activation barrier for changing their behavior in the classroom. It shared effective teaching practices, influenced faculty attitudes towards teaching, and empowered them to change their own behavior in the classroom. This success is succinctly captured by a participant's feedback:

The program has strengthened my interest in teaching effectiveness and stimulated me to continue thinking about innovations in teaching.

\section{Acknowledgements}

Support for the faculty development program described here was provided by the College of Engineering at U-M.

\section{References}

1. Dancy, M. \& Henderson, C. (2010). Pedagogical practices and instructional change of physics faculty. American Journal of Physics, 78, 1056-1063.

2. Felder, R. M., Brent, R., \& Prince, M. J. (2011). Engineering instructional development: Programs, best practices, and recommendations. Journal of Engineering Education, 100(1), 89-122.

3. Finelli, C. J., \& Daly, S. R. (2011, Oct). Teaching practices of engineering faculty: Self-reported behavior and actual practice. Proceedings of the 2011 International Research in Engineering Education Symposium, Madrid, Spain.

4. Finelli, C. J., Pinder-Grover, T., \& Wright, M. C. (2011). Consultations on teaching. Using student feedback for instructional improvement. In C. E. Cook \& M. L. Kaplan (Eds.), Advancing the culture of teaching at a research university: How a teaching center can make a difference. pp. 65-79. Sterling VA: Stylus Publishing.

5. Finelli, C. J., Richardson, K. M., \& Daly, S. R. (2013). Factors that influence faculty motivation to adopt effective teaching practices in engineering. Proceedings of the 2013 ASEE Annual Conference \& Exposition, Atlanta, GA. 
6. Handelsman, J., Ebert-May, D., Beichner, R., Bruns, P., Chang, A., DeHaan, R., Gentile, J., Lauffer, S., Stewart, J., Tilghman, S. M., \& Wood, W. B. (2004). Scientific teaching. Science, 304(5670), 521-522.

7. Hora, M. T. (2012). Organizational factors and instructional decision-making: A cognitive perspective. The Review of Higher Education, 35(2), 207-235.

8. Hora, M., Ferrare, J., \& Anderson, C. (2009). Structured observation protocol for instruction in institutions of higher education. Madison, WI: University of Wisconsin-Madison, Wisconsin Center for Education Research.

9. Light, G., Calkins, S., Luna, M., \& Drane, D. (2009). Assessing the impact of a year-long faculty development program on faculty approaches to teaching. International Journal of Teaching and Learning in Higher Education, 20(2). 168-181.

10. Murray, H. G. (1985). Classroom teaching behaviors related to college teaching effectiveness. In J.G. Donald \& A.M. Sullivan (Eds.), Using Research to Improve Teaching. San Francisco: Jossey-Bass.

11. Prince, M. (2004). Does active learning work? A review of the research. Journal of Engineering Education, 93(3), 223-246.

12. Smith, K. A., Sheppard, S. D., Johnson, D. W., \& Johnson, R. T. (2005). Pedagogies of engagement: Classroom-based practices (Cooperative learning and Problem-based learning). Journal of Engineering Education, 94 (1), 87-102.

13. Trigwell, K., \& Prosser, M. (2004). Development and use of the Approaches to Teaching Inventory. Educational Psychology Review, 16(44), 409-424.

14. Wlodkowski, R. J. (1999). Enhancing adult motivation to learn: A comprehensive guide for teaching all adults, 2nd Ed. New York, NY: John Wiley and Sons. 